\title{
Comparison of numerical and standard sarnat grading using the NICHD and SIBEN methods
}

\author{
Brian H. Walsh $\mathbb{D}^{1,2^{凶}}$, Chelsea Munster $\mathbb{D}^{2}$, Hoda El-Shibiny ${ }^{2}$, Edward Yang ${ }^{3}$, Terrie E. Inder ${ }^{2}$ and Mohamed El-Dib (iD ${ }^{2}$ \\ (c) The Author(s) 2021
}

OBJECTIVE: The NICHD and SIBEN assessments are adapted from the Sarnat grade, and used to determine severity of neonatal encephalopathy (NE). We compare NICHD and SIBEN methods, and their ability to define a minimum threshold associated with significant cerebral injury.

STUDY DESIGN: Between 2016 and 2019, 145 infants with NE (77-mild; 65-moderate; 3-severe) were included. NICHD and SIBEN grade and numerical scores were assigned. Kappa scores described agreement between methods, and ROC curves their ability to predict MR injury.

RESULTS: Good agreement existed between grading systems $(K=0.86)$. SIBEN defined more infants as moderate, and less as mild, than NICHD $(p<0.001)$. Both numerical scores were superior to standard grades in predicting MR injury.

CONCLUSION: Despite good agreement between methods, SIBEN defines more infants as moderate NE. Both numerical scores were superior to standard grade, and comparable to each other, in defining a minimum threshold for cerebral injury. Further assessment contrasting their predictive ability for long-term outcome is required.

Journal of Perinatology (2022) 42:328-334; https://doi.org/10.1038/s41372-021-01180-w

\section{INTRODUCTION}

The Sarnat grade, and subsequent modifications of it, are used to define the severity of Neonatal Encephalopathy (NE), and are predictive of cerebral injury as well as long-term outcome [1, 2]. However to be used appropriately a grade of encephalopathy cannot be assigned for several days using these scoring systems, long after the window for treatment with therapeutic hypothermia (TH) has elapsed. In recognition of this, early grading systems derived from the Sarnat grade have been developed, to allow timely assessment of $\mathrm{NE}$ and determination of $\mathrm{TH}$ eligibility. However, recent studies have shown that infants with milder encephalopathy may still demonstrate an increased risk for cerebral injury challenging clinicians to review the systematic neurological assessment used to evaluate these infants [3-5]. The goal for clinicians should now be to determine how best to identify the minimum threshold at which injury occurs.

The NICHD system is commonly used in North America to define severity of encephalopathy into one of three grades, mild, moderate or severe $[6,7]$. However severity of encephalopathy is not as simple as these grades. In the recent PRIME study, Chalak et al. applied a numerical value (0-3) to each of the six domain of the NICHD system in their cohort of infants with mild NE $[7,8]$. Using this method they demonstrated that there is a range of severity within each grade of encephalopathy, and that the distinction between grades is often vague, with overlapping values when a numerical score is applied. For example infants with mild NE could score from 1 to 10, while moderate NE ranged from 6 to 14 , and severe NE from 9 to 18. Perhaps unsurprisingly infants with mild NE that had higher NICHD scores $(\geq 5)$ were those at risk of neuro-developmental impairment.

The SIBEN system has recently been described as an alternate method of early assessment $[7,8]$. The SIBEN exam assesses the same domains as the NICHD system, defining the severity of encephalopathy as mild, moderate or severe. However unlike the NICHD system, SIBEN does not weight either seizures, or the subdomains of the primitive reflexes and autonomic system. Each of these findings is counted as an equal domain. Therefore although the same exam is performed, SIBEN requires findings in $\geq 3$ out of ten domains, while NICHD requires findings in $\geq 3$ out of six domains to categortize an infant as moderate or severe NE. It is likely that this would result in some infants being defined as moderate NE by SIBEN, but mild by NICHD, although the two methods have not been previously compared to confirm this.

In this manuscript our aim was to assess the variation of encephalopathy severity described by numerical scoring systems applied to both the NICHD and SIBEN grading systems, and to determine if either system (using both grades and numerical scores) was superior at detecting a minimum threshold associated with significant cerebral perturbation or injury, defined by the development of electrographic seizures, abnormal electroencephalography (EEG) background at $24 \mathrm{~h}$, or significant MRI injury.

\section{METHODOLOGY}

This is a retrospective analysis of infants with NE who underwent TH between January 2016 and May 2019 in the Brigham and

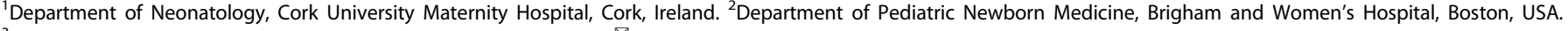
${ }^{3}$ Department of Radiology, Boston Children's Hospital, Boston, USA. ${ }^{\circledR}$ email: brianhenry.walsh@ucc.ie 
Women's Hospital, a large tertiary level NICU. The need to initiate $\mathrm{TH}$ was determined by the clinician, and based upon an adaptation of standard criteria which we have previously described $[5,9]$. Specifically these criteria state and infant must be (1) >34 weeks gestation, and (2) have a risk for perinatal asphyxia defined as one of the following; sentinel event prior to delivery (e.g. uterine rupture); or prolonged resuscitation at birth (on-going intermittent positive pressure ventilation or intubated at $10 \mathrm{~min}$ after birth); or $10 \mathrm{~min}$ Apgar score $\leq 5$; or $\mathrm{pH} \leq 7.1$ from the cord gas or postnatal gas within the first $1 \mathrm{~h}$ of life; or base deficit $\geq 10 \mathrm{mEq} / \mathrm{L}$ from the cord gas or postnatal gas within the first $1 \mathrm{~h}$ of life; and (3) evidence of seizures or clinical encephalopathy. In assessing $\mathrm{pH}$ and base deficit, while the umbilical arterial values are most reflective of the fetal state [10], the worst value from any cord or postnatal sample in the first hour of life was used to determine TH eligibility in accordance with the methodology of the TH RCTs and international recommendations [11-16]. Available samples included umbilical arterial samples in 120 cases, umbilical venous in 124 cases (paired umbilical arterial and venous in 117), and postnatal blood gas samples in 140. Of the worst samples reported-116 were umbilical arterial ( 4 arterial samples were excluded as the results appeared erroneous when compared to the cord venous and or postnatal samples), 6 were umbilical venous (cases with no umbilical arterial sample present), and 23 were postnatal samples. All infants had a neurological exam performed by both an attending neonatologist and a pediatric neurologist, within the first $6 \mathrm{~h}$ of life prior to initiation of $\mathrm{TH}$. For inclusion in this analysis, in addition to assigning an overall grade of NE, all components and sub-components of the NICHD exam had to be documented in-full within the medical records. Institutional Review Board approval was obtained to conduct this analysis from the Partners Human Research Committee.

Demographic, clinical and laboratory data, including maternal prenatal history, delivery history, and postnatal history until discharge, were collected from the medical records. All infants included in this cohort underwent $\mathrm{TH}$, had multichannel EEG during cooling and had an MRI scan following re-warming. The continuous EEGs were placed as soon as possible after the decision to initiate $\mathrm{TH}$, and were maintained through-out the $72 \mathrm{~h}$ of cooling and re-warming. For this analysis the clinical neurophysiologist report from the first $24 \mathrm{~h}$ of age were used to define the EEG grade of encephalopathy. The presence of electrographic seizures at any point during the EEG monitoring was recorded.

\section{NICHD grade and score}

The NICHD grade assesses six neurological domains; level of consciousness, spontaneous activity, tone, posture, primitive reflexes (two sub-domains assessed independently, with the worst score providing the global grade for primitive reflexessuck, and Moro reflex), and autonomic activity (three sub-domains assessed independently, with the worst score providing the global grade for autonomic activity, pupillary reaction, heart rate and respirations) (Supplementary Table 1). Each of the six domains is defined as normal, mild, moderate or severe. A global grade of NE is then determined; an infant is scored as mild if they have at least one domain consistent with mild, but do not meet criteria for moderate or severe NE; an infant is scored as moderate if they have three or more domains consistent with moderate or severe $\mathrm{NE}$, but more domains are moderate than severe; and they are scored severe if they have three or more domains consistent with moderate or severe $\mathrm{NE}$, but more domains are severe than moderate. Seizures are not included in the six domains, however the presence of seizures and findings of NE automatically define the grade of NE as at least moderate.

The NICHD score described by Chalak et al. assigned a numerical score from 0 to 3 (consistent with normal to severe findings respectively) to each of the six domains. The score across all domains was then added and a global numerical score provided [7]. Seizures were not included in this system.

\section{SIBEN grade and score}

The SIBEN grade assesses the same exam components as the NICHD system; level of consciousness, spontaneous activity, tone, posture, suck reflex, Moro reflex, pupillary reaction, heart rate and respirations (Supplementary Table 1) [6]. However it does not weight the sub-domains of the autonomic system or primitive reflexes, and each is treated as an equal independent domain. Additionally seizures are counted within the score, as an independent domain. Of note seizures do not automatically assign a global grade of moderate NE. Therefore there are ten domains in the SIBEN system, each of which is defined as normal, mild, moderate or severe. A global grade of NE is then determined; an infant is scored as mild if they have at least three domains consistent with mild, but do not meet criteria for moderate or severe NE; an infant is scored as moderate if they have three or more domains consistent with moderate or severe $\mathrm{NE}$, but more domains are moderate than severe; and they are scored severe if they have three or more domains consistent with moderate or severe $\mathrm{NE}$, but more domains are severe than moderate. Of note, seizures were included in defining grade if they occurred in the first $6 \mathrm{~h}$ of life. If they occurred at a later point, the grade was not revised due to the development of seizures.

A numerical score has not previously been published for the SIBEN system. We have therefore proceeded in a similar fashion to that described by Chalak et al. assigning a numerical score from 0 to 3 (consistent with normal to severe findings respectively) to each of the ten domains. The score across all domains was then added and a global numerical score provided.

\section{Magnetic resonance imaging}

All infants had at least one cranial MRI performed within the first week of life. The clinical teams caring for the infant determined the timing of these MRI studies and whether a second MRI was required. Only the initial MRI scans are reported here due to the variability in a second scan being performed. All scans were performed on a 3-T Siemens scanner (Siemens, Erlangen, Germany). The standard clinical imaging protocol included T1, $\mathrm{T} 2$, and diffusion weighted imaging. The images for this study were analyzed independently by a pediatric neuroradiologist, and neonatologist (EY, TI), who were blinded to the clinical grades of encephalopathy. The presence and type of any MRI abnormalities were detailed. Analysis of the pattern and severity of brain injury was classified according to the grading system developed by Barkovich et al., which has been extensively validated in NE using both conventional and diffusion weighted sequences [17]. A score of $\geq 2$ in the deep nuclear gray matter, or a score of $\geq 3$ in a watershed pattern, was considered consistent with moderatesevere MRI injury.

\section{Statistical analyses}

Statistical analysis was performed using PASW statistics 18.0. Nonparametric data were reported as median values with interquartile range (IQR) or range as specified in the text, and comparisons performed using the Mann-Whitney $U$ test or Kruskal-Wallis $H$ Test, as appropriate. The Chi Squared test was used when comparing proportions. Agreement between grades was described using Kappa values.

Logistic regression analysis was performed to assess the strength of association between the individual methods of assessing NE severity (covariate) and the development of moderate-severe MR cerebral injury (dependent variable). Additionally receiver operator curves were generated to determine their ability to predict moderate-severe MR cerebral injury. The sensitivity and specificity were determined from the ROC curves. We wished to determine the benefits of these methods to be used 
as a screening tool to detect the minimum threshold for significant injury. Therefore the cut-off value described was that associated with the optimum sensitivity. Statistical significance was taken as $p<0.05$, and a Bonferroni correction was applied for post-hoc sub-group analysis.

\section{RESULTS}

During the study period there were 170 infants with NE who received $\mathrm{TH}$ in our center. Twenty five infants were excluded as they had incomplete documentation of the NE assessment subdomains (Of those exclude 11 were documented as having mild $\mathrm{NE}, 9$ moderate $\mathrm{NE}, 2$ with severe $\mathrm{NE}$, and 2 did not have any grade of NE assigned). This left a study population of 145 infants. Demographic details are provided in Table 1.

Table 1. Clinical and demographic details for cohort.

\begin{tabular}{|c|c|c|c|}
\hline NICHD grade & Mild NE $(n=77)$ & $\begin{array}{l}\text { Moderate NE ( } n \\
=65)\end{array}$ & $\begin{array}{l}\text { Severe NE }(n= \\
\text { 3) }\end{array}$ \\
\hline Gestational age & $39.14(1.58)$ & $38.83(1.77)$ & 40.7 (1.12) \\
\hline Birth weight & 3232 (535) & 3085 (532) & $3656(390)$ \\
\hline Sex (female) & 34 (44\%) & 25 (38.5\%) & $1(33 \%)$ \\
\hline \multicolumn{4}{|c|}{ Method of delivery } \\
\hline SVD & $23(30 \%)$ & $23(35 \%)$ & \\
\hline Instrumental & $10(14 \%)$ & $10(15 \%)$ & $2(66 \%)$ \\
\hline Em-LSCS & 39 (51\%) & 31 (48\%) & $1(33 \%)$ \\
\hline EI-LSCS & $4(5 \%)$ & $1(2 \%)$ & \\
\hline $\mathrm{pH}^{\mathrm{a}}$ & $7.02(6.80-7.35)$ & $7.04(6.80-7.35)$ & $6.94(6.80-7.23)$ \\
\hline Base deficit $^{a}$ & $12.9(10.6-14.9)$ & $13(10.2-13.4)$ & $17.6(16.4-18.8)$ \\
\hline Apgar $5 \mathrm{~min}$ & $7(2-9)$ & $6(0-8)$ & $0(0-2)$ \\
\hline Apgar $10 \mathrm{~min}$ & $8(5-10)$ & $7(0-10)$ & $2(1-3)$ \\
\hline $\begin{array}{l}\text { Invasive } \\
\text { ventilation }\end{array}$ & $13(17 \%)$ & $23(35 \%)$ & $3(100 \%)$ \\
\hline $\begin{array}{l}\text { Electrical } \\
\text { seizure }\end{array}$ & 7 (9\%) & $9(14 \%)$ & $3(100 \%)$ \\
\hline Seizure 1 st $6 \mathrm{~h}$ & $0(0 \%)$ & $1(2 \%)$ & $0(0 \%)$ \\
\hline \multicolumn{4}{|l|}{ EEG background } \\
\hline Normal & $14(18 \%)$ & $13(20 \%)$ & 0 \\
\hline Mild & $52(68 \%)$ & $31(48 \%)$ & 0 \\
\hline Moderate & 7 (9\%) & $10(15 \%)$ & 0 \\
\hline Severe & 0 & $1(2 \%)$ & $3(100)$ \\
\hline Length of stay & $7(4-42)$ & $8(2-35)$ & $18(9-38)$ \\
\hline
\end{tabular}

aLowest value from either an umbilical cord blood sample or any postnatal blood sample $<1 \mathrm{~h}$ after birth. (Sample used-umbilical cord artery $n=$ 116, umbilical vein $n=6$, and postnatal sample $n=23$ ).

\section{NICHD grade and numerical score}

Of the 145 infants, based upon the NICHD criteria, 77 infants had mild NE, 65 had moderate, and 3 had severe NE. There was a significant difference in the median NICHD Numerical score between those with mild or moderate NE in our cohort, 5 (range $1-7)$ vs. 8 (range 6-13) vs. 15 (range 15-18), $p<0.001$, respectively. Figure 1 demonstrates that there is a transition in scores between mild and moderate NE in the 6-10 range as expected. As can be seen in our data, although possible for a mild infant to have a score of 9 or 10, no mild NE had a score above 7 . Of our cohort, $16 \%$ [12] of mild NE, and $42 \%$ (27) of infants with moderate NE had a score within the overlapping range of 6-7.

\section{SIBEN grade and numerical score}

Utilising the SIBEN system 68 had mild NE, 74 had moderate NE, and 3 had severe NE. Similar to the NICHD score, there was a significant difference in the median SIBEN scores between grades, with a median score of 4 (range 1-7) vs. 8 (range 6-17) vs. 19 (range 18-22), $p<0.001$, respectively. Again there was overlap between mild and moderate NE grades, with 13\% $(n=9)$ and $38 \%$ $(n=28)$, of the SIBEN mild and moderate NE infants, respectively, having a score from 6 to 7 (Fig. 1).

\section{Comparison of NICHD and SIBEN grade}

The NICHD and SIBEN grading systems agreed in $92 \%$ of cases $(134 / 145)$, with a Kappa value of $0.86(p<0.001)$. Despite this high rate of agreement, the differences between the assigned grades was statistically significant $(p<0.001)$, with the SIBEN system having fewer infants defined as mild NE, and more as moderate $\mathrm{NE}$, compared to the NICHD system. Details on all infants for whom there was a discrepancy in the grades is provided in Table 2. As can be seen, the majority are infants with mild NE by the NICHD but moderate using SIBEN, with NICHD scores of $<6$. The only infant that was moderate per NICHD but mild per SIBEN, had hypertonia in addition to two other moderately abnormal domains. Hypertonia is not included on the SIBEN score, hence the reason for the discrepancy in grades.

\section{Association with EEG}

By $24 \mathrm{~h}$ of age, the background EEG was normal in 27 infants, mildly encephalopathic in 83, moderately encephalopathic in 17 and severely encephalopathic in 4. EEG data was not available on 14 infants. Nineteen infants developed electrographic seizures. The median age of seizure onset was $13 \mathrm{~h}$ (IQR 7-17 h). The distribution of background EEG grade, and electrographic seizures for each of the grading systems is demonstrated in Fig. 2.

In comparing the systems, there was no difference in the background EEG grade for those classified as mild per NICHD vs. mild per SIBEN $(p=0.95)$, moderate per NICHD vs. moderate per SIBEN $(p=0.97)$ or severe per NICHD vs. severe per SIBEN $(p=1)$
A

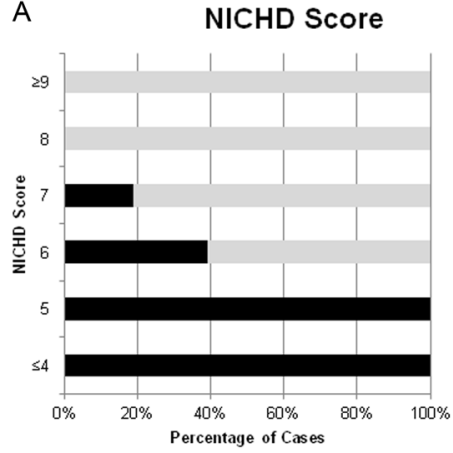

B

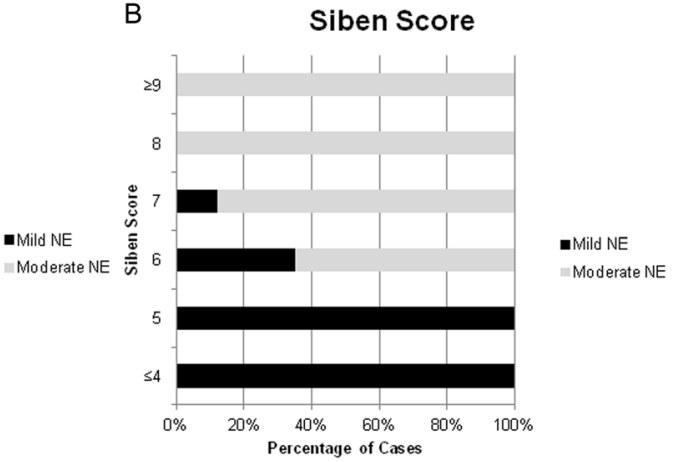

Fig. 1 Association between Grade of Encephalopathy and Numerical Score. A Frequency of NICHD Grade associated with specific NICHD Numerical Score. B Frequency of SIBEN Grade associated with specific SIBEN Numerical Score. 


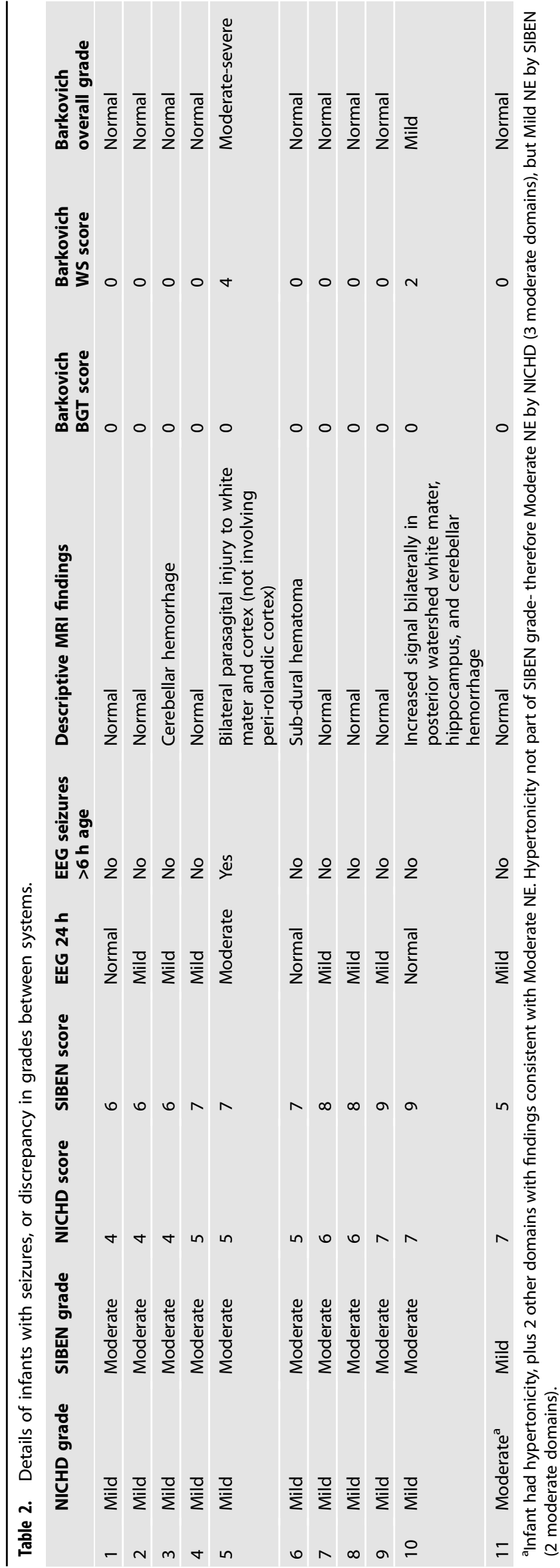

NE. Seven infants that were graded as mild per NICHD versus 6 who were mild per SIBEN developed electrographic seizures $(p=$ 0.59 ). Similarly there was no difference in frequency of electrographic seizures between those classified using the NICHD vs. SIBEN system as moderate $(p=0.99)$, or severe $(p=1)$ NE.

\section{Association of early neurological assessment with cerebral MRI}

The median age at MRI scan was 4 (range 0-15) days. 104 (72\%) MRls had no findings consistent with ischemic injury on the Barkovich system, while 41 (28\%) did demonstrate ischemic injury. Of these 41 with identified injury, 15 had injury within the Basal Ganglia and/or Thalamus, and 34 had injury in a Watershed distribution. Twenty four (17\%) infants MRIs' were consistent with mild injury, and $17(12 \%)$ had moderate or severe cerebral injury on MRI. The distribution of cerebral MR injury stratified by each of the grading systems is demonstrated in Fig. 2. Although some variation between the grading systems, there was no significant difference in the frequency or severity of MR cerebral injury comparing those classified using the NICHD vs. SIBEN grade as mild $(p=0.97)$, moderate $(p=0.92)$ or severe $(p=1)$ NE.

Logistic regression was used to compare the strength of association between, while receiver operator curves were generated to assess predictive ability for, each of the methods of neurological assessment (NICHD grade, SIBEN grade, NICHD score, and SIBEN score) and a moderate-severely abnormal MRI (Table 3). As can be seen each method significantly predicted the development of a moderate-severely abnormal MRI. The NICHD and SIBEN numerical scoring systems had a greater association, and were superior at predicting a moderate-severely abnormal MRI than the categorical grades using either system. There was no difference in the strength of association between the NICHD and SIBEN numerical scores. For both of the scoring systems a cut-off of $\geq 4$ was the optimum score for identifying those with a moderate-severe cerebral injury on MRI (Table 3).

\section{DISCUSSION}

In this study we report that while there is good agreement between the NICHD and SIBEN systems, there are subtle but important differences. The disagreements between the two occur at the threshold between mild and moderate NE. This reflects the fact that within an individual grade of NE there is a range of severity. The use of numerical scoring systems highlights this issue, documenting the range within each grade, and the considerable overlap in the burden of neurological disturbance observed between grades. We report that both the NICHD and SIBEN grading systems detected those at increased risk of significant cerebral injury, however numerical scoring systems for either method were superior for detecting the minimum threshold of increased risk. The numerical scoring systems were comparable, and for both we found a cut-off of $\geq 4$ provided the optimum sensitivity for detecting a significant cerebral insult.

There was over $90 \%$ agreement between the NICHD and SIBEN grades assigned. The differences in the grades are primarily due to the differing methods of synthesizing the same exam findings $[6,7]$. Unlike the NICHD system, SIBEN does not weight the autonomic system (heart rate, respiration, pupils) and primitive reflexes (suck, Moro) by category, but counts each of the five sub-domains equally. Therefore for NICHD the worst finding from the constituent subdomains are used to assign a single grade to both the autonomic system and primitive reflexes, but for SIBEN 5 equal grades are defined. While SIBEN's approach is in keeping with the original Sarnat method, the NICHD's approach would ensure a broader neurological disturbance is present when defining moderate or severe NE, which would reduce the numbers of cases. This reflects our findings, that the SIBEN system assigned several infants as moderate $\mathrm{NE}$, that were classified as mild NE by the NICHD system. 
Row A NICHD Grade and Seizures

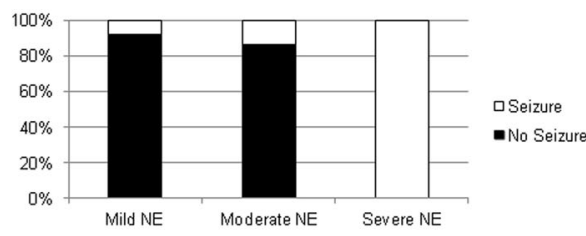

Row B NICHD Grade and EEG grade

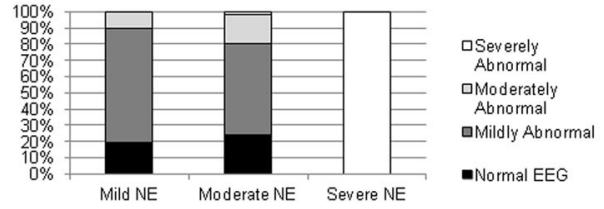

Row $C \quad$ NICHD Grade and MRI

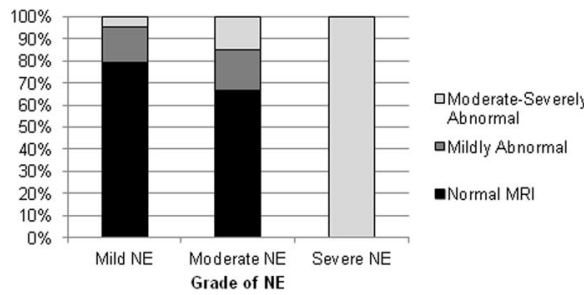

SIBEN Grade and Seizures

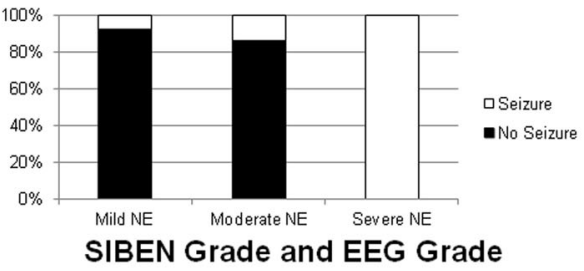

Severely

口Moderately

Abnormal
a Mildly Abnormal

- Normal EEG

Fig. 2 Association between NICHD and SIBEN grades and short-term outcomes. A A It demonstrates association of NICHD and SIBEN grade with presence of Seizures. B It demonstrates association of NICHD and SIBEN grade with multi-channel EEG grade. C It demonstrates association of NICHD and SIBEN grade with MRI cerebral injury.

Table 3. Ability of each grading and scoring system to predict Moderate-Severe Hypoxic Ischemic injury on MRI.

To predict a moderate-severe MRI

\begin{tabular}{|c|c|c|c|c|c|c|c|c|}
\hline & $r^{2}$ & $p$ & AUC & $p$ & $\mathrm{Cl}$ & Cut-off & Sensitivity & Specificity \\
\hline NICHD grade & 0.221 & $<0.001$ & 0.71 & 0.006 & $0.57-0.84$ & Moderate & 76 & 57 \\
\hline SIBEN grade & 0.227 & $<0.001$ & 0.71 & 0.005 & $0.58-0.84$ & Moderate & 78 & 49 \\
\hline NICHD score & 0.267 & $<0.001$ & 0.79 & $<0.001$ & $0.67-0.90$ & $\geq 4$ & 100 & 9 \\
\hline SIBEN score & 0.260 & $<0.001$ & 0.78 & $<0.001$ & $0.66-0.89$ & $\geq 4$ & 100 & 10 \\
\hline
\end{tabular}

In this manuscript, we contrast two numerical scoring systems. The NICHD score was recently described by Chalak et al. and the second numerical score based upon the SIBEN grade has not previously been published. Alternate numerical scores have been described for prognosis following NE, however unlike the NICHD and SIBEN methods, these scores were designed to predict longterm prognosis over several days, rather than to be used in the first hours of life to identify encephalopathy and determine treatment eligibility $[18,19]$. Furthermore, several of the domains described in these scores differ between those of the NCIHD and SIBEN methods, and include assessments of fontanel, grasp, and feeding, which may not be practical within the first $6 \mathrm{~h}[18,19]$. Potentially due to such issues, they have been reported to poorly identify those eligible for TH [20]. In contrast we report that both numerical scoring systems were superior to the standard grade of encephalopathy for predicting which infants would develop significant cerebral perturbation and injury.

Numerical scoring systems have several potential advantages compared to standard grading systems. Chalak et al. use the NICHD score to demonstrate the range of severity that exists among those with mild NE [7]. Unlike the work of Chalak et al., our data includes infants with mild, moderate and severe NE. This has allowed us to demonstrate the range in severity, and overlap, that exists particularly among those with mild and moderate NE. Using, the NICHD score $16 \%$ of mild and $42 \%$ of moderate grades had overlapping scores in the 6-7 range (NICHD score mild 1-10, moderate 6-14). The overlap was slightly less using the SIBEN score, with $13 \%$ of mild and $38 \%$ of moderate cases having overlapping scores. Being able to assess the severity within an individual grade of NE is necessary to highlight those at greater risk outside of the classical TH eligibility, such as those with mild $\mathrm{NE}$ at greater risk of injury.

An additional benefit of numerical scoring systems is that they reduce the impact of several of the more subjective components of either the NICHD or SIBEN exam. For example a weak suck can be defined as a mild or moderately encephalopathic finding using either assessment method. There is similar overlap in findings between mild and moderate encephalopathy for assessment of tone, and spontaneous activity. These overlapping findings blur the distinction between grades, and allow some subjectivity in assessment. It must be recognized that this blurring of findings between grades is not necessarily inaccurate, and in fact likely reflects the true nature of this disease which is a continuum rather than distinct isolated grades [1]. However the subjectivity that they introduce can be problematic when so much emphasis is placed on the grade of NE for determining eligibility for TH. The use of a numerical scoring system rather than grade of NE to determine risk of injury would limit the impact of these more subjective components of the exam, as for example with the NICHD system a difference in 1 or 2 points when being assessed on a continuous scale from 0 to 18 would have less of an impact on the over-all synthesis. 
The primary limitation of this study is that there is no long-term neuro-developmental outcome data available for this cohort. Although this is a limitation, cerebral injury on MRI is highly associated with 2 year neuro-developmental outcome [21], with the presence of moderate-severe injury being highly predictive of significant neuro-developmental impairment. Furthermore it must be recognized that the SIBEN grade itself has not yet been validated for neuro-developmental outcome. Given it's similarities with the NICHD grade, and the similar results for predicting a significantly abnormal MRI that we report here, it is likely that it will have similar predictive abilities as the NICHD grade for long-term outcome, but this is yet to be validated. An additional limitation is that this was a secondary analysis. As such it is retrospective in nature, with the potential for unidentified confounders impacting the results. However the fact that this is a large cohort of infants with $N E$, including 142 infants with mild or moderate $\mathrm{NE}$, somewhat reduces such risk. Lastly, this study was limited by the fact that our local guidelines include providing $\mathrm{TH}$ to those with milder grades of encephalopathy $[5,9]$. As such all infants included in the current analysis underwent $\mathrm{TH}$. It is unknown if $\mathrm{TH}$ reduces the burden of injury with mild $N E$, however there is evidence of improved MR spectroscopy in these infants [11,22]. Twenty eight percent of infants in our study demonstrated cerebral injury on MRI. This is consistent with recent literature, which has demonstrated that the majority of those with moderate or severe NE that undergo TH have a normal MRI scan [23]. Rollins et al. recently reported that only $17 \%$ of those with moderate or severe NE have a significant MR injury following $\mathrm{TH}$ [24]. Therefore treatment with TH may have adjusted the optimum cut-offs for the numerical scores to identify injury. This will need to be studied further in infants that do not receive $\mathrm{TH}$.

In conclusion we found a strong agreement between the SIBEN and NICHD systems. The SIBEN did define more infants as moderate NE than the NICHD system did. Numerical scores based upon both systems highlighted the range of neurological disturbance that exists within individual grades of NE. Likely for this reason the numerical scores were superior at identifying infants at increased risk of developing seizures, significant EEG abnormalities and MRI injury. We propose that screening for $\mathrm{TH}$ eligibility should be determined using numerical scores rather than grade of NE. This would aid in identifying those most at risk of injury, particularly for those at the threshold between grades who do not currently receive therapy. To adopt such an approach further work is required to validate the minimum threshold for injury among those who do not receive $\mathrm{TH}$.

\section{REFERENCES}

1. Sarnat HB, Sarnat MS. Neonatal encephalopathy following fetal distress. A clinical and electroencephalographic study. Arch Neurol. 1976;33:696-705.

2. Robertson C, Finer N. Term infants with hypoxic-ischemic encephalopathy: outcome at 3.5 years. Dev Med Child Neurol. 1985;27:473-84.

3. Conway JM, Walsh BH, Boylan GB, Murray DM. Mild hypoxic ischaemic encephalopathy and long term neurodevelopmental outcome-a systematic review. Early Hum Dev. 2018;120:80-7.

4. Finder M, Boylan GB, Twomey D, Ahearne C, Murray DM, Hallberg B. Two-year neurodevelopmental outcomes after mild hypoxic ischemic encephalopathy in the era of therapeutic hypothermia. JAMA Pediatr. 2020;174:48-55.

5. Walsh BH, Neil J, Morey J, Yang E, Silvera MV, Inder TE, et al. The frequency and severity of magnetic resonance imaging abnormalities in infants with mild neonatal encephalopathy. J Pediatr. 2017;187:26-33.e1.

6. Perez JM, Golombek SG, Sola A. Clinical hypoxic-ischemic encephalopathy score of the Iberoamerican Society of Neonatology (Siben): a new proposal for diagnosis and management. Rev Assoc Med Bras. 2017;63:64-9.

7. Chalak LF, Adams-Huet B, Sant'Anna G. A total sarnat score in mild hypoxicischemic encephalopathy can detect infants at higher risk of disability. J Pediatr. 2019;214:217-21.e1.

8. Chalak LF, Nguyen KA, Prempunpong C, Heyne R, Thayyil S, Shankaran S, et al. Prospective research in infants with mild encephalopathy identified in the first six hours of life: neurodevelopmental outcomes at 18-22 months. Pediatr Res. 2018;84:861-8.

9. https://www.rmf.harvard.edu/Clinician-Resources/Guidelines-Algorithms/2016/ neonatalencephaopathy- download [CRICO guidelines].

10. Westgate J, Garibaldi JM, Greene KR. Umbilical cord blood gas analysis at delivery: a time for quality data. Br J Obstet Gynaecol. 1994;101:1054-63.

11. Shankaran S, Laptook AR, Ehrenkranz RA, Tyson JE, McDonald SA, Donovan EF, et al. Whole-body hypothermia for neonates with hypoxic-ischemic encephalopathy. N Engl J Med. 2005;353:1574-84.

12. Gluckman PD, Wyatt JS, Azzopardi D, Ballard R, Edwards AD, Ferriero DM, et al. Selective head cooling with mild systemic hypothermia after neonatal encephalopathy: multicentre randomised trial. Lancet. 2005;365:663-70.

13. Azzopardi DV, Strohm B, Edwards AD, Dyet L, Halliday HL, Juszczak E, et al Moderate hypothermia to treat perinatal asphyxial encephalopathy. $\mathrm{N}$ Engl J Med. 2009;361:1349-58.

14. Jacobs SE, Morley CJ, Inder TE, Stewart MJ, Smith KR, McNamara PJ, et al. Wholebody hypothermia for term and near-term newborns with hypoxic-ischemic encephalopathy: a randomized controlled trial. Arch Pediatr Adolesc Med. 2011;165:692-700.

15. Therapeutic Hypothermia for Neonatal Encephalopathy. A BAPM Framework for Practice. 2020. https://www.bapm.org/resources/237-therapeutic-hypothermiafor-neonatal-encephalopathy.

16. Papile LA, Baley JE, Benitz W, Cummings J, Carlo WA, Eichenwald E, et al. Hypothermia and neonatal encephalopathy. Pediatrics. 2014;133:1146-50.

17. Barkovich AJ, Hajnal BL, Vigneron D, Sola A, Partridge JC, Allen F, et al. Prediction of neuromotor outcome in perinatal asphyxia: evaluation of MR scoring systems. AJNR Am J Neuroradiol. 1998;19:143-9.

18. Thompson CM, Puterman AS, Linley LL, Hann FM, van der Elst CW, Molteno CD, et al. The value of a scoring system for hypoxic ischaemic encephalopathy in predicting neurodevelopmental outcome. Acta Paediatr. 1997;86:757-61.

19. Miller SP, Latal B, Clark H, Barnwell A, Glidden D, Barkovich AJ, et al. Clinical signs predict 30-month neurodevelopmental outcome after neonatal encephalopathy. Am J Obstet Gynecol. 2004;190:93-9.

20. Lally PJ, Price DL, Pauliah SS, Bainbridge A, Kurien J, Sivasamy N, et al. Neonatal Encephalopathic Cerebral Injury in South India Assessed by Perinatal Magnetic Resonance Biomarkers and Early Childhood Neurodevelopmental Outcome. Plos ONE. 2014;9:e87874.

21. Rutherford M, Ramenghi LA, Edwards AD, Brocklehurst $P$, Halliday $H$, Levene $M$, et al. Assessment of brain tissue injury after moderate hypothermia in neonates with hypoxic-ischaemic encephalopathy: a nested substudy of a randomised controlled trial. Lancet Neurol.2010;9:39-45.

22. Montaldo P, Lally PJ, Oliveira V, Swamy R, Mendoza J, Atreja G, et al. Therapeutic hypothermia initiated within $6 \mathrm{~h}$ of birth is associated with reduced brain injury on MR biomarkers in mild hypoxic-ischaemic encephalopathy: a non-randomised cohort study. Arch Dis Child Fetal Neonatal Ed. 2019;104:F515-F20.

23. Shankaran S, Barnes PD, Hintz SR, Laptook AR, Zaterka-Baxter KM, McDonald SA, et al. Brain injury following trial of hypothermia for neonatal hypoxic-ischaemic encephalopathy. Arch Dis Child-Fetal Neonatal Ed. 2012;97:F398-F404.

24. Rollins N, Booth T, Morriss MC, Sanchez P, Heyne R, Chalak L. Predictive value of neonatal mri showing no or minor degrees of brain injury after hypothermia. Pediatr Neurol. 2014;50:447-51.

\section{FUNDING}

Open Access funding provided by the IReL Consortium.

\section{COMPETING INTERESTS}

The authors declare no competing interests.

\section{ADDITIONAL INFORMATION}

Supplementary information The online version contains supplementary material available at https://doi.org/10.1038/s41372-021-01180-w.

Correspondence and requests for materials should be addressed to B.H.W.

Reprints and permission information is available at http://www.nature.com/reprints

Publisher's note Springer Nature remains neutral with regard to jurisdictional claims in published maps and institutional affiliations. 
Open Access This article is licensed under a Creative Commons Attribution 4.0 International License, which permits use, sharing, adaptation, distribution and reproduction in any medium or format, as long as you give appropriate credit to the original author(s) and the source, provide a link to the Creative Commons license, and indicate if changes were made. The images or other third party material in this article are included in the article's Creative Commons license, unless indicated otherwise in a credit line to the material. If material is not included in the article's Creative Commons license and your intended use is not permitted by statutory regulation or exceeds the permitted use, you will need to obtain permission directly from the copyright holder. To view a copy of this license, visit http://creativecommons. org/licenses/by/4.0/.

(c) The Author(s) 2021 